\title{
EFEITO DE QUEIMADAS SOBRE A ESTRUTURA E COMPOSIÇÃO DA COMUNIDADE VEGETAL LENHOSA DO CERRADO SENTIDO RESTRITO EM CALDAS NOVAS, GO ${ }^{1}$
}

\author{
Sérgio de Faria Lopes ${ }^{2}$, Vagner Santiago do Vale ${ }^{2}$ e Ivan Schiavini ${ }^{3}$
}

\begin{abstract}
RESUMO - Considerando que o regime de queima é um dos principais fatores que alteram a estrutura e composição de espécies de uma comunidade vegetal, este trabalho teve como objetivo analisar o efeito do fogo na composição florística e estrutura da vegetação lenhosa de Cerrado sentido restrito no Parque Estadual da Serra de Caldas Novas (PESCAN), Goiás, submetida a diferentes regimes de queima. Foram utilizadas duas áreas de Cerrado sentido restrito: uma delas submetida ao fogo em 2002 e 2006 e outra sem a passagem do fogo nessas duas épocas. Em cada área foram estabelecidas 25 parcelas de 20 × $20 \mathrm{~m}$, sendo incluídas no estudo todas as árvores $\left(C_{30} \geq 15 \mathrm{~cm}\right)$. As duas áreas estudadas apresentaram composição florística similar, evidenciada pelo Coeficiente de Sorensen, o qual encontrou $84 \%$ de similaridade na composição florística entre as áreas. Entretanto, a estrutura da vegetação apresentou diferenças relevantes, evidenciada pelo Indice de Similaridade de Bray Curtis, o qual obteve valor de 0,67 , que foi refletido por mudanças na estrutura das comunidades estudadas. A área queimada apresentou menor número de indivíduos, de espécies, menor valor de área basal e, por conseguinte, do Índice de Diversidade de Shannon (H') e Equabilidade (J'). Nesse sentido, provavelmente a frequência das queimadas ocorridas na área não foram suficientes para evidenciar o efeito do fogo no processo de alteração na composição das espécies lenhosas. Entretanto, o fogo exerceu papel relevante na modificação da estrutura da vegetação.
\end{abstract}

Palavras-chave: Fogo, Parque Estadual da Serra de Caldas Novas e fitossociologia.

\section{EFFECT OF FIRE ON THE STRUCTURE AND FLORISTIC COMPOSITION OF A CERRADO SENSU STRICTO AREA IN CALDAS NOVAS, GO}

\begin{abstract}
Considering that the regimes of burning modify important factors of the structure and composition of the species of a plant community, the objective of this work was to analyze the effect of burning on the floristic composition and structure of the wood vegetation of the cerrado sensu stricto at the Parque Estadual da Serra de Caldas Novas (PESCAN), Goiás, submitted to different times of burning. Two areas had been used of Cerrado sensu stricto: one was submitted to burning in 2002 and 2006 and the other area did not suffer burning in the same period. For each area, 25 parcels of $20 \times 20 \mathrm{~m}$ were established and all the wood flora $(C 30 \geq 15 \mathrm{~cm})$ was included in the study. Both areas studied presented similar floristic composition, evidenced by the Sorensen Coefficient, which found $84 \%$ of similarity in the floristic composition between the areas. However, the vegetation structure presented relevant differences, evidenced by the Similarity's Index of Bray Curtis, which found a value of 0,67. This value was reflected by changes in the studied communities' structure. The burnt area presented a smaller number of individuals and species, a smaller value for the basal area and, therefore, the Shannon Diversity Index ( $\left.H^{\prime}\right)$ and Evenness ( $\left.J^{\prime}\right)$. Therefore, the frequency and intensity of the burnings in the area had not been enough to evidence their effects on the process of alteration in the composition of the wood species. However, the fire exerted a relevant role in the modification of the vegetation structure.
\end{abstract}

Keywords: Burning, Parque Estadual da Serra de Caldas Novas and phytossociology.

\footnotetext{
${ }^{1}$ Recebido em 11.10.2007 e aceito para publicação em 29.05.2009.

${ }^{2}$ Pós-graduaçãoemEcologiaeConservaçãodeRecursos NaturaisdaUniversidadeFederal deUberlândia(UFU).E-mail: <lopeserginho@yahoo.com.br>.

${ }^{3}$ Instituto de Biologia da UFU. E-mail: <schiavini@ufu.br>.
} 


\section{INTRODUÇÃO}

As formas fisionômicas mais comuns do Cerrado caracterizam-se por possuir um estrato rasteiro bastante desenvolvido, constituído principalmente por gramíneas e um estrato lenhoso não muito denso, onde as copas das árvores não formam um dossel contínuo (RIBEIRO e WALTER, 1998).

Na estação seca, as gramíneas, em sua maioria, estão inativas, e a maior parte de sua biomassa aérea seca morre, o que favorece a ocorrência de incêndios (KLINK e SOLBRIG, 1996). Embora a vegetação lenhosa do Cerrado apresente características adaptativas ao fogo, as queimadas durante a estação seca podem resultar em mudanças relevantes na estrutura e composição florística da vegetação do que as queimadas provocadas na estação chuvosa devido, principalmente, à intensidade delas (COUTINHO, 1990; EITEIN, 1994).

A baixa exigência nutricional da vegetação nativa do Cerrado confere às fitofisionomias savânicas alta resiliência quanto ao restabelecimento da cobertura vegetal depois do fogo. Entretanto, queimadas muito frequentes podem significar empobrecimento do ecossistema como um todo quanto ao estoque de nutrientes essenciais e uma eventual redução de biomassa total, sobretudo da camada arbórea e arbustiva (MIRANDA et al., 2004).

Nesta perspectiva, em áreas onde o fogo é frequente, uma progressiva redução da diversidade e simplificação da estrutura da comunidade pode ocorrer, tornando a fisionomia gradualmente mais aberta (HOFFMANN e MOREIRA, 2002). Dessa forma, a densidade do estrato arbóreo estaria determinada mais pela habilidade das espécies em sobreviver ao fogo e regenerar, do que pela própria competição pelos recursos ambientais (DURIGAN et al., 1994).

O fogo é um fator de perturbação no bioma Cerrado, que geralmente determina a estrutura da vegetação e sua composição. Ou seja, a sobrevivência de organismos ao fogo é determinada por suas características anatômicas, fisiológicas e comportamentais, além das características ambientais pós-fogo. As modificações na composição das comunidades vegetais pós-queima dependerão, fundamentalmente, da resposta de cada espécie ao fogo, tendo por influência a densidade, os padrões de alocação e forma de crescimento de cada espécie (BOND e WILGEN, 1996).

R. Árvore, Viçosa-MG, v.33, n.4, p.695-704, 2009
Considerando que o regime de queima é um dos principais fatores que alteram a estrutura e composição de espécies de uma comunidade vegetal (GILL, 1975), este trabalho teve como objetivo analisar o efeito do fogo na composição florística e estrutura da comunidade vegetal lenhosa de um Cerrado sentido restrito no Parque Estadual da Serra de Caldas Novas, GO, submetida a diferentes regimes de queima. Este estudo visa ampliar o conhecimento sobre a influência do fogo na determinação da composição florística e na estrutura de comunidades vegetais lenhosas, bem como responder algumas a questões envolvidas: a) qual a influência do fogo na composição e estrutura da comunidade vegetal dessa área de Cerrado? b) a intensidade e frequência de queimadas são fatores mais relevantes na determinação da composição ou da estrutura da comunidade? Buscou-se responder a essas questões, tendo em vista a hipótese de que o fogo é um fator influenciador direto na formação da estrutura da comunidade vegetal; porém, a influência do fogo na mudança da composição florística é um processo dependente da intensidade e frequência de queimadas.

\section{MATERIAL E MÉTODOS}

O estudo foi realizado no Parque Estadual da Serra de Caldas Novas (PESCAN), entre os vértices de coordenadas $17^{\circ} 47^{\prime} 13^{\prime \prime}$ sul e $48^{\circ} 40^{\prime} 12^{\prime \prime}$ ' oeste. Na Serra de Caldas Novas, encontra-se uma vegetação típica do bioma Cerrado, com um mosaico de fitofisionomias que se estendem desde Cerrado sentido restrito, campo sujo e campo limpo, principalmente no platô da Serra, Cerrado e campo rupestre nas encostas com afloramentos rochosos, algumas manchas de cerradão, veredas em locais úmidos e matas de galeria próximo aos córregos e nascentes nos vales da encosta (MAGNAGO et al., 1983; NOVAES et al., 1983).

A pluviosidade média anual da região é de 1.500 $\mathrm{mm}$, distribuídos irregularmente, com um verão quente e chuvoso e um inverno seco, sendo a temperatura média anual de $23^{\circ} \mathrm{C}$. Essas características climáticas se enquadram no tipo Aw (KÖPPEN, 1948). No platô da Serra, encontra-se, principalmente, a predominância de Cerrado sentido restrito, ocorrendo, em geral, sobre vários tipos de latossolos (MAGNAGO et al., 1983; NOVAES et al., 1983).

A coleta de dados foi realizada durante o mês de outubro de 2006. A área de estudo abrange 2 ha no platô do Parque, a cerca de 990 m de altitude. Para 
isso, foram utilizadas duas áreas de Cerrado sentido restrito: uma delas submetida ao fogo em 2002 e com uma reincidência em agosto de 2006 e outra sem a passagem do fogo nessas duas épocas. As duas áreas estão separadas por uma estrada de terra, considerada como barreira física entre elas, funcionando como aceiro e não permitindo a passagem do fogo nas duas épocas citadas.

Em cada área foram estabelecidas 25 parcelas sistemáticas de $20 \times 20 \mathrm{~m}$, resultando uma área amostral total de $10.000 \mathrm{~m}^{2}$ (1 ha). Nessas parcelas foram identificados todos os indivíduos arbóreos vivos, com diâmetro igual ou superior a $5 \mathrm{~cm}$, registrado a 0,30 $\mathrm{m}$ de altura do solo, segundo o protocolo da Rede de Parcelas Permanentes dos Biomas Cerrado e Pantanal (FELFILI et al., 2005). Também foram anotados os dados relativos à altura dos indivíduos. A classificação das famílias foi feita de acordo com o sistema Angiosperm Phylogeny Group II (SOUZA e LORENZI, 2005).

Os parâmetros fitossociológicos de densidade, dominância e frequência relativas e o valor de importância (VI) (MATTEUCCI e COLMA, 1982) foram analisados utilizando-se o programa FITOPAC 1 (SHEPHERD, 1995). Para a avaliação da diversidade alfa, foram utilizados o índice de diversidade de Shannon (H') e o de equabilidade de Pielou (J') (BROWER e ZAR, 1984). Também, foi analisada a distribuição dos indivíduos da comunidade em classes diamétricas. Para a análise da estrutura comunitária foram estabelecidos intervalos de classes de diâmetro e altura da comunidade em $5 \mathrm{~cm}$ e $1,5 \mathrm{~m}$, respectivamente, conforme utilizado por Nascimento et al. (2004).

As duas áreas de Cerrado sentido restrito estudadas foram comparadas qualitativamente (presença e ausência de espécies), utilizando-se o coeficiente de similaridade de Sørensen e, quantitativamente (número de indivíduos por espécie), o índice de Bray Curtis (HORN, 1966). Os resultados dos índices variam de 0 a 1 , sendo uma similaridade considerada alta se o valor for maior que 0,5 e, quanto mais próximo esse valor de 1 , maior a similaridade. Para essa análise foi utilizado o Programa FITOPAC 1 (SHEPHERD, 1995).

\section{RESULTADOS E DISCUSSÃO}

\subsection{Composição florística e estrutura da vegetação}

As famílias mais importantes foram as mesmas em ambas as áreas, modificando apenas as posições no VI (valor de importância), devido às diferenças na abundância e frequência dos indivíduos amostrados. Segundo Bridgewater et al. (2004), a flora do Cerrado é composta comumente por um pequeno grupo de famílias, porém, no nível de espécies, essa flora é bastante diversificada. Vochysiaceae e Bignoniaceae foram as famílias com os maiores valores de VI na área não queimada, enquanto na área queimada as famílias Bignoniaceae e Fabaceae apresentaram os maiores VI.

A família Vochysiaceae teve o maior VI na área não queimada, assim como em outros trabalhos (FELFILI et al., 1993; SILVA et al., 2002), devido à contribuição decorrente da área basal de espécies como Qualea parviflora e $Q$. grandiflora. Muitas espécies de Vochysiaceae são típicas acumuladoras de alumínio (HARIDASAN e ARAÚJO, 1998), e isso lhes proporciona vantagem competitiva, permitindo o desevolvimento em solos ácidos do Cerrado (FELFILI e SILVA JÚNIOR, 1992; BALDUINO et al., 2005). Entretanto, a família Bignoniaceae teve maior representativiadade em ambas as áreas, devido à alta densidade de Tabebuia aurea.

As famílias Fabaceae e Vochysiaceae estão entre as mais importantes em ambas as áreas de estudo, confirmando os dados encontrados em outras áreas de Cerrado (FELFILI et al., 1993; FIEDLER et al., 2004; SANTOS e VIEIRA, 2005). Entretanto, a composição de espécies foi diferente dos registros de Silva et al. (2002), o que reforça a hipótese de heterogeneidade do Cerrado (RATTER e DARGIE, 1992). Uma clara evidência disso é o elevado valor de importância atribuída à Tabebuia aurea, espécie que normalmente possui baixa densidade em áreas de Cerrado (RATTER et al., 2003). Já Caryocar brasiliense apresentou baixa densidade neste estudo, em ambas as áreas, ao passo que é normalmente apontada como uma espécie característica de Cerrado sentido restrito do PESCAN (SILVA et al., 2002). A amostragem deste estudo foi bem representativa e característica da fitofisionomia estudada, uma vez que $100 \%$ das espécies de maior VI também foram amostradas em pelo menos $50 \%$ das 376 áreas analisadas por Ratter et al. (2003). Como também $100 \%$ das espécies de maior VI em ambas as áreas estão presentes na lista das 121 espécies dominantes da flora do Cerrado, segundo Bridgewater et al. (2004).

Tabebuia aurea foi a espécie como maior Valor de Importância (VI) nas duas áreas levantadas (Tabela 1), representando $31 \%$ e $19,1 \%$ de todos os indivíduos amostrados na área queimada e não queimada, respectivamente. As duas áreas apresentaram pequena

R. Árvore, Viçosa-MG, v.33, n.4, p.695-704, 2009 
mudança na composição das espécies com maiores VI, devido a diferenças na abundância e dominância (Tabela 1). As 10 espécies mais importantes nas duas áreas estão presentes na Tabela 2. Foram encontradas 56 espécies comuns nas duas áreas. Além disso, um grupo de espécies em comum formado por Aspidosperma tormentosum, Bowdichia virgilioides, Kielmeyera coriaceae, Ouratea hexasperma, Stryphnodendron adstringens e Tabebuia aurea apresentaram ampla distribuição em ambas as áreas, sendo amostradas pelo menos em 20 parcelas.
Apesar de Qualea parviflora apresentar menor número de indivíduos em relação a T. aurea, na área não queimada essa espécie apresentou maior dominância, devido ao maior diâmetro do caule. $\mathrm{Na}$ área queimada, T. aurea apresentou os maiores valores em todos os parâmetros relativos. As espécies Machaerium acutifolium, Enterolobium gummiferum, Pouteria torta, Guapira noxia, Vatairea macrocarpa e Andira paniculata apresentaram apenas um indivíduo na área queimada, enquanto na área queimada 11 espécies mostraram apenas um indivíduo.

Tabela 1 - Lista dos parâmetros fitossociológicos, em ordem decrescente de IVI da área queimada, das espécies presentes nas duas áreas de Cerrados sentido restrito do Parque Estadual da Serra de Caldas Novas (PESCAN), GO

Table 1 - List of phytosociological parameters in two cerrado sensu stricto sites at Parque Estadual da Serra de Caldas Novas (PESCAN), GO

\begin{tabular}{|c|c|c|c|c|c|c|c|c|c|c|}
\hline \multirow[t]{2}{*}{ Espécies } & \multicolumn{2}{|c|}{$\mathrm{N} \mathrm{I}$} & \multicolumn{2}{|c|}{ DR } & \multicolumn{2}{|c|}{ DoR } & \multicolumn{2}{|c|}{ FR } & \multicolumn{2}{|c|}{ IVI } \\
\hline & AQ & ANQ & $\mathrm{AQ}$ & ANQ & $\mathrm{AQ}$ & ANQ & $\mathrm{AQ}$ & ANQ & $\mathrm{AQ}$ & ANQ \\
\hline Tabebuia aurea (Silva Manso) & 482 & 320 & 30,62 & 19,08 & 27,94 & 14,80 & 4,75 & 4,12 & 21,1 & 12,7 \\
\hline \multicolumn{11}{|l|}{ Benth. \& Hook. f. ex S. Moore } \\
\hline Kielmeyera coriacea Mart. \& Zucc & 119 & 128 & 7,56 & 7,63 & 6,53 & 5,58 & 4,75 & 4,30 & 6,3 & 5,8 \\
\hline Bowdichia virgilioides Kunth & 58 & 35 & 3,68 & 2,05 & 5,91 & 5,28 & 4,37 & 4,82 & 4,7 & 0,0 \\
\hline Aspidosperma tomentosum Mart. & 67 & 50 & 4,26 & 2,98 & 4,04 & 2,70 & 4,18 & 3,44 & 4,2 & 3,0 \\
\hline Dalbergia miscolobium Benth. & 24 & 9 & 1,52 & 0,54 & 6,55 & 1,77 & 2,66 & 1,37 & 3,6 & 1,2 \\
\hline Kielmeyera grandiflora (Wawra) Saddi & 59 & 16 & 3,75 & 0,95 & 2,76 & 0,93 & 3,61 & 1,55 & 3,4 & 1,1 \\
\hline Stryphnodendron adstringens (Mart.) Coville & 50 & 65 & 3,18 & 3,88 & 3,24 & 3,54 & 3,61 & 3,78 & 3,3 & 3,7 \\
\hline Strichnos pseudo-quina A. St.-Hil. & 37 & 9 & 2,35 & 0,54 & 3,22 & 0,72 & 3,61 & 1,20 & 3,1 & 0,8 \\
\hline Piptocarpha rotundifolia (Less.) Baker & 42 & 38 & 2,67 & 2,27 & 2,60 & 2,97 & 3,80 & 3,09 & 3,0 & 2,8 \\
\hline Ouratea hexasperma (A. St.-Hil.) Baill. & 46 & 70 & 2,92 & 4,17 & 2,99 & 4,18 & 3,04 & 3,78 & 3,0 & 4,0 \\
\hline Palicourea rigida Kunth & 46 & 45 & 2,92 & 2,68 & 1,85 & 1,18 & 3,99 & 3,44 & 2,9 & 2,4 \\
\hline Styrax ferrugineus Nees \& Mart. & 41 & 22 & 2,60 & 1,31 & 2,58 & 1,27 & 3,23 & 2,58 & 2,8 & 1,7 \\
\hline Byrsonima coccolobifolia Kunth & 40 & 15 & 2,54 & 0,89 & 1,72 & 0,50 & 3,23 & 1,72 & 2,5 & 1,0 \\
\hline Psidium firmum $\mathrm{O}$. Berg & 21 & 3 & 1,33 & 0,18 & 2,24 & 0,13 & 3,04 & 0,52 & 2,2 & 0,3 \\
\hline Brosimum gaudichaudii Trécul & 36 & 23 & 2,29 & 1,37 & 1,25 & 0,56 & 2,66 & 2,58 & 2,1 & 1,5 \\
\hline Eremanthus glomerulatus Less. & 33 & 53 & 2,10 & 3,16 & 1,28 & 1,25 & 2,66 & 3,09 & 2,0 & 2,5 \\
\hline Qualea grandiflora Mart. & 14 & 64 & 0,89 & 3,82 & 3,15 & 9,31 & 1,90 & 3,26 & 2,0 & 5,5 \\
\hline Erythroxylum suberosum A. St.-Hil. & 29 & 45 & 1,84 & 2,68 & 0,99 & 1,36 & 2,85 & 2,92 & 1,9 & 2,3 \\
\hline Machaerium opacum Vogel & 17 & 3 & 1,08 & 0,18 & 1,56 & 0,28 & 2,28 & 0,52 & 1,6 & 0,3 \\
\hline Psidium aerugineum O. Berg & 17 & 4 & 1,08 & 0,24 & 1,15 & 0,11 & 2,47 & 0,52 & 1,6 & 0,3 \\
\hline Licania humilis Cham. \& Schltdl. & 19 & 44 & 1,21 & 2,62 & 1,50 & 2,96 & 1,90 & 3,09 & 1,5 & 2,9 \\
\hline Diospyros burchellii DC & 17 & 5 & 1,08 & 0,30 & 1,53 & 0,10 & 1,90 & 0,86 & 1,5 & 0,4 \\
\hline Tabebuia ochracea (Cham.) Standl. & 17 & 3 & 1,08 & 0,18 & 1,48 & 0,05 & 1,90 & 0,52 & 1,5 & 0,2 \\
\hline Aspidosperma macrocarpon Mart. & 20 & 10 & 1,27 & 0,60 & 0,75 & 0,28 & 2,28 & 1,03 & 1,4 & 0,6 \\
\hline Qualea multiflora Mart. & 22 & 27 & 1,40 & 1,61 & 1,13 & 1,11 & 1,71 & 1,89 & 1,4 & 1,5 \\
\hline Vochysia cinnamomea Pohl & 22 & 65 & 1,40 & 3,88 & 0,94 & 2,02 & 1,33 & 3,61 & 1,2 & 3,2 \\
\hline Byrsonima verbascifolia (L.) DC. & 20 & 19 & 1,27 & 1,13 & 0,83 & 1,10 & 1,52 & 1,72 & 1,2 & 1,3 \\
\hline Annona crassiflora Mart. & 12 & 28 & 0,76 & 1,67 & 0,68 & 2,73 & 1,71 & 3,09 & 1,1 & 2,5 \\
\hline Davilla elliptica A. St.-Hil & 18 & 0 & 1,14 & 0,00 & 0,67 & 0,00 & 1,33 & 0,00 & 1,0 & 0,0 \\
\hline Connarus suberosus Planch. & 15 & 9 & 0,95 & 0,54 & 0,42 & 0,19 & 1,71 & 1,37 & 1,0 & 0,7 \\
\hline Tocoyena formosa (Cham. \& Schltdl.) & 11 & 10 & 0,70 & 0,60 & 0,32 & 0,22 & 1,71 & 0,17 & 0,9 & 0,7 \\
\hline
\end{tabular}


Tabela 1 - Cont

Table 1 - Cont.

\begin{tabular}{|c|c|c|c|c|c|c|c|c|c|c|}
\hline \multirow[t]{2}{*}{ Espécies } & \multicolumn{2}{|c|}{$\mathrm{N} \mathrm{I}$} & \multicolumn{2}{|c|}{ DR } & \multicolumn{2}{|c|}{ DoR } & \multicolumn{2}{|c|}{ FR } & \multicolumn{2}{|c|}{ IVI } \\
\hline & $\mathrm{AQ}$ & ANQ & $\mathrm{AQ}$ & ANQ & $\mathrm{AQ}$ & ANQ & $\mathrm{AQ}$ & ANQ & $\mathrm{AQ}$ & ANQ \\
\hline Stryphnodendron polyphyllum Mart. & 13 & 20 & 0,83 & 1,19 & 0,28 & 0,40 & 1,14 & 2,23 & 0,7 & 1,3 \\
\hline Dimorphandra mollis Benth. & 8 & 2 & 0,51 & 0,12 & 0,58 & 0,03 & 1,14 & 0,34 & 0,7 & 0,2 \\
\hline Acosmium dasycarpum (Vogel) Yakovlev & 9 & 8 & 0,57 & 0,48 & 0,69 & 0,22 & 0,95 & 0,86 & 0,7 & 0,5 \\
\hline Erythroxylum tortuosum Mart. & 7 & 25 & 0,44 & 1,49 & 0,29 & 0,51 & 1,33 & 2,41 & 0,7 & 1,5 \\
\hline Vochysia rufa Mart. & 8 & 2 & 0,51 & 0,12 & 0,56 & 0,03 & 0,95 & 0,17 & 0,7 & 0,1 \\
\hline Plenckia populnea Reissek & 7 & 2 & 0,44 & 0,12 & 0,41 & 0,05 & 0,95 & 0,34 & 0,6 & 0,2 \\
\hline Qualea parviflora Mart. & 6 & 181 & 0,38 & 10,79 & 0,26 & 15,00 & 1,14 & 3,61 & 0,6 & 9,8 \\
\hline Byrsonima pachyphylla A. Juss. & 6 & 24 & 0,38 & 1,43 & 0,34 & 0,90 & 0,95 & 2,75 & 0,6 & 1,7 \\
\hline Casearia sylvestris $\mathrm{Sw}$ & 8 & 2 & 0,51 & 0,12 & 0,17 & 0,04 & 0,76 & 0,34 & 0,5 & 0,2 \\
\hline Pouteria ramiflora (Mart.) Radlk. & 4 & 55 & 0,25 & 3,28 & 0,73 & 6,56 & 0,38 & 3,26 & 0,5 & 4,4 \\
\hline Hymenaea stigonocarpa Mart. ex Hayne & 3 & 5 & 0,19 & 0,30 & 0,43 & 0,54 & 0,38 & 0,86 & 0,3 & 0,6 \\
\hline Tabebuia insignis (Miq.) Sandwith & 3 & 0 & 0,19 & 0,00 & 0,11 & 0,00 & 0,57 & 0,00 & 0,3 & 0,0 \\
\hline Hancornia speciosa Gomes & 3 & 9 & 0,19 & 0,54 & 0,22 & 0,71 & 0,38 & 1,37 & 0,3 & 0,9 \\
\hline Caryocar brasiliense Cambess. & 2 & 17 & 0,13 & 1,01 & 0,27 & 1,45 & 0,38 & 1,55 & 0,3 & 1,3 \\
\hline Salvertia convallariodora A. St.-Hil. & 1 & 2 & 0,06 & 0,12 & 0,38 & 0,08 & 0,19 & 0,34 & 0,2 & 0,2 \\
\hline Roupala montana Aubl. & 2 & 21 & 0,13 & 1,25 & 0,06 & 1,10 & 0,38 & 1,72 & 0,2 & 1,4 \\
\hline Psidium sp. & 2 & 0 & 0,13 & 0,00 & 0,07 & 0,00 & 0,19 & 0,00 & 0,1 & 0,0 \\
\hline Sclerolobium paniculatum Vogel & 1 & 2 & 0,06 & 0,12 & 0,10 & 0,12 & 0,19 & 0,34 & 0,1 & 0,2 \\
\hline Kielmeyera rubriflora Cambess. & 1 & 6 & 0,06 & 0,36 & 0,05 & 0,39 & 0,19 & 0,69 & 0,1 & 0,5 \\
\hline Rourea induta Planch. & 1 & 0 & 0,06 & 0,00 & 0,03 & 0,00 & 0,19 & 0,00 & 0,1 & 0,0 \\
\hline Aegiphila klotzkiana Cham. & 1 & 4 & 0,06 & 0,24 & 0,03 & 0,17 & 0,19 & 0,52 & 0,1 & 0,3 \\
\hline Eriotheca gracilipes (K. Schum.) A. Robyns & 1 & 0 & 0,06 & 0,00 & 0,03 & 0,00 & 0,19 & 0,00 & 0,1 & 0,0 \\
\hline Miconia ferruginata DC. & 1 & 0 & 0,06 & 0,00 & 0,02 & 0,00 & 0,19 & 0,00 & 0,1 & 0,0 \\
\hline Neea theifera Oerst. & 1 & 2 & 0,06 & 0,12 & 0,03 & 0,07 & 0,19 & 0,17 & 0,1 & 0,1 \\
\hline Vatairea macrocarpa (Benth.) Ducke & 1 & 1 & 0,06 & 0,06 & 0,02 & 0,03 & 0,19 & 0,17 & 0,1 & 0,1 \\
\hline Vochysia tucanorum Mart. & 1 & 0 & 0,06 & 0,00 & 0,03 & 0,00 & 0,19 & 0,00 & 0,1 & 0,0 \\
\hline $\begin{array}{l}\text { Agonandra brasiliensis Miers ex } \\
\text { Benth. \& Hook. f. }\end{array}$ & 1 & 0 & 0,06 & 0,00 & 0,02 & 0,00 & 0,19 & 0,00 & 0,1 & 0,0 \\
\hline Himatanthus obovatus (Müll. Arg.) Woodson & 1 & 9 & 0,06 & 0,54 & 0,02 & 0,26 & 0,19 & 1,20 & 0,1 & 0,7 \\
\hline Andira paniculata Benth. & 0 & 1 & 0,00 & 0,06 & 0,00 & 0,02 & 0,00 & 0,17 & 0,0 & 0,1 \\
\hline Enterolobium gummiferum (Mart.) J.F. Macbr. & 0 & 1 & 0,00 & 0,06 & 0,00 & 0,09 & 0,00 & 0,17 & 0,0 & 0,1 \\
\hline $\begin{array}{l}\text { Eriotheca pubescens (Mart. \& Zucc.) } \\
\text { Schott. \& Endl. }\end{array}$ & 0 & 8 & 0,00 & 0,48 & 0,00 & 0,77 & 0,00 & 1,03 & 0,0 & 0,8 \\
\hline Erythroxylum deciduum A. St.-Hil. & 0 & 2 & 0,00 & 0,12 & 0,00 & 0,03 & 0,00 & 0,17 & 0,0 & 0,1 \\
\hline Guapira noxia (Netto) Lundell & 0 & 1 & 0,00 & 0,06 & 0,00 & 0,04 & 0,00 & 0,17 & 0,0 & 0,1 \\
\hline Lafoensia pacari A. St.-Hil. & 0 & 11 & 0,00 & 0,66 & 0,00 & 0,57 & 0,00 & 0,69 & 0,0 & 0,6 \\
\hline Machaerium acutifolium Vogel & 0 & 1 & 0,00 & 0,06 & 0,00 & 0,13 & 0,00 & 0,17 & 0,0 & 0,1 \\
\hline Myrcia uberavensis $\mathrm{O}$. Berg & 0 & 2 & 0,00 & 0,12 & 0,00 & 0,05 & 0,00 & 0,17 & 0,0 & 0,1 \\
\hline Pouteria torta (Mart.) Radlk. & 0 & 1 & 0,00 & 0,06 & 0,00 & 0,08 & 0,00 & 0,17 & 0,0 & 0,1 \\
\hline Salacia crassifolia (Mart. ex Schult.) G. Don & 0 & 2 & 0,00 & 0,12 & 0,00 & 0,03 & 0,00 & 0,34 & 0,0 & 0,2 \\
\hline Schefflera macrocarpa (Seem) D.C. Frodin & 0 & 11 & 0,00 & 0,66 & 0,00 & 0,34 & 0,00 & 1,55 & 0,0 & 0,8 \\
\hline
\end{tabular}

$\mathrm{NI}=$ número de indivíduos; $\mathrm{DR}=$ densidade relativa; $\mathrm{DoR}=$ dominância relativa; $\mathrm{FR}=$ frequência relativa; $\mathrm{IVI}=$ índice de valor de importância; $\mathrm{AQ}=$ área queimada; e ANQ = área não queimada.

$\mathrm{NI}=$ number of individuals; DR = relatives densities; DoR = relatives dominance; FR = relatives frequency; IVI = Valor of Importance Index; $\mathrm{AQ}=$ burnt sites; and $\mathrm{ANQ}=$ site not burnt.

Foram encontrados, na área queimada, 1.574 indivíduos pertencentes a 59 espécies e 29 famílias, enquanto na área não queimada se observaram 1.677 indivíduos pertencentes a 62 espécies e 28 famílias (Tabela 3). Em relação à área basal, a área queimada $\left(\operatorname{com~} 11,24 \mathrm{~m}^{2}\right)$ apresentou valor menor do que a área não queimada $\left(14,38 \mathrm{~m}^{2}\right)$. A baixa exigência nutricional da vegetação nativa do Cerrado confere ao ecossistema alta resiliência quanto ao restabelecimento da cobertura vegetal depois do fogo (MIRANDA et al., 2004). Entretanto, para esses autores queimadas frequentes podem significar empobrecimento do ecossistema como 
um todo quanto ao estoque de nutrientes essenciais e eventual redução de biomassa total, sobretudo da camada arbórea e arbustiva. A maior área basal observada na área não queimada deveu-se, em grande parte, a um maior número de indivíduos amostrados de Qualea parviflora (181) e Q. grandiflora (64), o que contribuiu para o aumento da área basal da comunidade nessa área, enquanto na área queimada foram amostrados apenas 6 e 14 indivíduos, respectivamente, daquelas duas espécies (Tabela 1).

A menor riqueza florística e alta dominância ecológica encontradas no Cerrado sentido restrito com a passagem do fogo refletiram em menores Índices de Diversidade e equabilidade (Tabela 3). Essa alta dominância ecológica encontrada na área queimada foi devida, em grande parte, à alta densidade de Tabebuia aurea .

\subsection{Distribuição em diâmetros}

As duas áreas estudadas apresentaram maior número de indivíduos com diâmetro menor do que $10 \mathrm{~cm}$ (Figura 1). A distribuição dos diâmetros da comunidade apresentou a forma de J-reverso, e 71\% dos indivíduos apresentaramse com diâmetro inferior a $10 \mathrm{~cm}$ na área queimada, enquanto na área não queimada esse valor foi de $65,4 \%$. O diâmetro máximo na área queimada encontrado foi de $41,7 \mathrm{~cm}$, pertencente a um indivíduo de Dalbergia miscolobium. Na área não queimada, esse valor foi de 34,7 cm para um indivíduo de Qualea grandiflora. No estudo realizado na APA do Paranoá, DF (ASSUNÇÃO e FELFILI, 2004), e em Nova Xavantina, MT (MARIMON JÚNIOR e HARIDASSAN, 2005), a distribuição diamétrica dos Cerrados amostrados também se apresentou em formato de J-reverso. A porcentagem de indivíduos com até $10 \mathrm{~cm}$ de diâmetro foi de $59 \%$ no estudo da APA do Paranoá (DF) e 50\% em Nova Xavantina (MT). Também foram encontrados valores semelhantes no estudo realizado na Chapada do Espigão Mestre do
São Francisco (FELFILI e SILVA JUNIOR, 2001). Cabe ressaltar que na área não queimada ocorreu maior número de indivíduos nas classes de maiores diâmetros. Isso pode indicar que indivíduos de maior porte podem não estar sofrendo mortalidade com a influência do fogo e, por conseguinte, modificando a estrutura da comunidade, como maior área basal total.

A maioria dos indivíduos amostrados na área queimada $(95,5 \%)$ apresentou diâmetro basal menor que $20 \mathrm{~cm}$, e somente duas espécies mostraram diâmetro basal maior que $30 \mathrm{~cm}$. Foram elas: Qualea grandiflora e Dalbergia miscolobium. Na área não queimada, 96,4\% dos indivíduos apresentaram diâmetro basal menor que $20 \mathrm{~cm}$. Entretanto, nessa área quatro espécies tiveram diâmetro maior do que $30 \mathrm{~cm}$ : Dalbergia miscolobium, Qualea parviflora, Bowdichia virgilioides e Qualea grandiflora. Entre as espécies com maior VI, Tabebuia aurea, Qualea parviflora, Bowdichia virgilioides, Qualea grandiflora e Dalbergia miscolobium, além de apresentarem os maiores diâmetros, foram as espécies com maiores valores de altura em ambas as áreas.

A altura média foi de 2,5 $\pm 1,1 \mathrm{~m}$ na área queimada, enquanto na área não queimada a altura média foi de 2,7 $\pm 1,3 \mathrm{~m}$. A distribuição das alturas em ambas as áreas foi bimodal, e 97,3\% dos indivíduos na área queimada e $94,8 \%$ na área não queimada tiveram alturas menores que $5 \mathrm{~m}$ (Figura 2). A maior percentagem (86,8\%) de altura está concentrada entre 0,5 e 3,5 m na área queimada e $82,3 \%$ na mesma classe na área não queimada. Apenas 10 indivíduos $(0,3 \%$ do total), pertencentes a cinco espécies, apresentaram altura superior a $8 \mathrm{~m}$. São elas: Tabebuia aurea, Qualea parviflora, Bowdichia virgilioides, Dalbergia miscolobium e Annona crassiflora. A maior altura medida foi de $9,5 \mathrm{~m}$, pertencente a um indivíduo amostrado de Qualea parviflora na área não queimada.

Tabela 2 - Lista das 10 espécies de maior IVI e o número de indivíduos nas duas áreas de Cerrado sentido restrito no PESCAN, GO Table 2 - List of ten species with greater IVI and number of individuals in two cerrado sensu stricto areas at PESCAN, GO

\begin{tabular}{lclc}
\hline Área queimada & Número de indivíduos & Área não queimada & Número de indivíduos \\
\hline Tabebuia aurea & 482 & Tabebuia aurea & 320 \\
Kielmeyera coriacea & 119 & Qualea parviflora & 181 \\
Bowdichia virgilioides & 58 & Kielmeyera coriacea & 128 \\
Aspidosperma tomentosum & 67 & Qualea grandiflora & 64 \\
Dalbergia miscolobium & 24 & Pouteria ramiflora \\
Kielmeyera grandiflora & 59 & Ouratea hexasperma \\
Stryphnodendron adstringens & 50 & Stryphnodendron adstringens \\
Strichnos pseudo-quina & 37 & Bowdichia virgilioides \\
Piptocarpha rotundifolia & 42 & Vochysia cinnamomea \\
Ouratea hexasperma & 46 & Aspidosperma tomentosum \\
\hline
\end{tabular}

R. Árvore, Viçosa-MG, v.33, n.4, p.695-704, 2009 
Tabela 3 - Parâmetros fitossociológicos das duas áreas de Cerrado sentido restrito do Parque Estadual da Serra de Caldas Novas (PESCAN), GO

Table 3 - Phytosociological parameters of the two cerrado sensu stricto sites at Parque Estadual da Serra de Caldas Novas (PESCAN), GO

\begin{tabular}{lcc}
\hline Parâmetros & $\begin{array}{c}\text { Área não } \\
\text { queimada }\end{array}$ & $\begin{array}{c}\text { Área } \\
\text { queimada }\end{array}$ \\
\hline Número de famílias & 28 & 29 \\
Número de espécies & 62 & 59 \\
Número de indivíduos & 1677 & 1574 \\
Área basal por hectare $\left(\mathrm{cm}^{2}\right)$ & 14,38 & 11,24 \\
Índice de Diversidade & 3,25 & 3,05 \\
Shannon (H') & & \\
Equabilidade & 0,79 & 0,48 \\
\hline
\end{tabular}

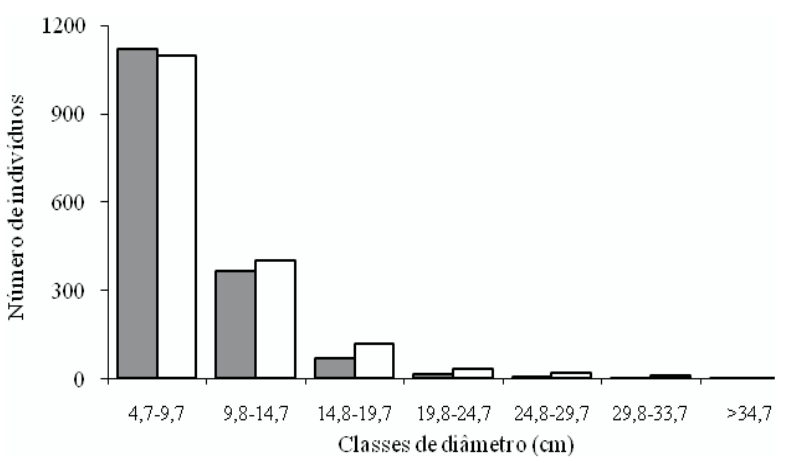

Figura 1 - Distribuição dos indivíduos por classe de diâmetro em duas áreas de Cerrado sentido restrito; área queimada (coluna cheia) e área não queimada (coluna vazia), no PESCAN, GO.

Figure 1 - Distribution of Individual per diameter classes in two cerrado sensu stricto sites; burnt site (full columns) and unburnt site (empty columns), in PESCAN, GO.

\subsection{Similaridade e influência do fogo}

As áreas estudadas apresentaram diversidade florística similar, evidenciada pelo Coeficiente de Sorensen, o qual encontrou $84 \%$ de similaridade na composição florística entre as áreas. Entretanto, apesar de as duas áreas amostradas demostrarem pequenas diferenças na composição florística e, principalmente, nas espécies de maior VI (Tabelas 1 e 2), a estrutura da vegetação apresentou diferenças relevantes, evidenciada pelo Índice de Similaridade de Bray Curtis, pelo qual foi encontrado valor de 0,67. Esse valor é refletido por mudanças na estrutura das comunidades estudadas. A área queimada teve menor número de indivíduos, de espécies, menor valor de área basal e, por conseguinte, do Índice de Diversidade de Shannon (H') e de Equabilidade (J'). Tal fato pode ser explicado pela maior frequência do fogo na área queimada, que podem influenciar a estabilidade do ambiente, em termos de resiliência e resistência em relação ao fogo (MIRANDA et al., 2004), pois esses regimes de queima em curtos espaços de tempo podem resultar em alta mortalidade, uma vez que as rebrotações podem não apresentar cascas espessas o suficiente ou por não terem atingido altura crítica para o escape do efeito direto do fogo.

Sambuichi (1991) observou que o número de espécies praticamente dobra depois de longos períodos de exclusão do fogo, convergindo para fisionomias mais fechadas. Na área de Cerrado que sofreu queimadas no PESCAN, verificou-se que a maior frequência de queimadas pode ter influenciado a menor densidade e tornado a vegetação mais baixa e aberta. Dessa forma, o fogo age como desbaste na vegetação lenhosa (FELFILI, 2000), enquanto a exclusão do fogo favorece o aumento em densidade e área basal do estrato lenhoso (HOFFMAN e MOREIRA, 2002) e a imigração de espécies mais sensíveis para a área, como observado por Henrique e Hay (2002).

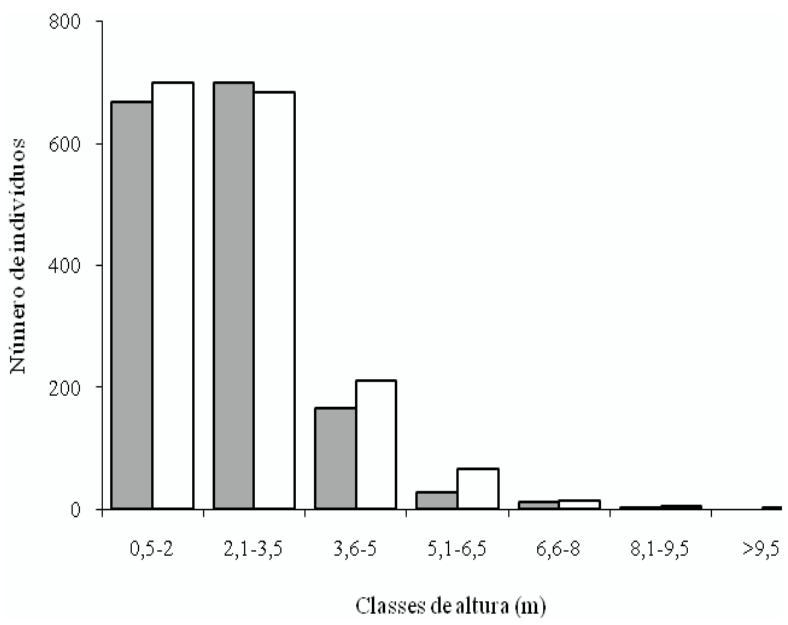

Figura 2 - Distribuição dos indivíduos por classe de altura em duas áreas de Cerrado sentido restrito; área queimada (coluna cheia) e área não queimada (coluna vazia), no PESCAN, GO

Figure 2 -Distribution of individuals per height classes in two cerrado sensu stricto sites; burnt site (full columns) and unburnt site (empty columns), in PESCAN, GO.

R. Árvore, Viçosa-MG, v.33, n.4, p.695-704, 2009 
A área não queimada apresentou $27,9 \%$ a mais em valor em área basal do que a área queimada. Isso se deve ao maior número de indivíduos de maior porte encontrados na área não queimada. Tal fato pode ser explicado pela maior intensidade de distúrbios ocorridos na área queimada, que podem estar influenciando a estabilidade do ambiente. Segundo Archer et al. (1996), as savanas são relativamente estáveis em termos de resiliência e resistência em relação a fogo, seca e herbivoria. No Tabela 1, verifica-se que Qualea grandiflora, Qualea parviflora, Pouteria ramiflora e Vochysia cinnamomea apresentaram valores de densidade bem superiores na área não queimada em relação à área queimada. Logo, sugere-se que essas espécies são mais sensíveis a distúrbios, principalmente ao fogo. No entanto, Dalbergia miscolobium, Kielmeyera grandiflora, Strichnos pseudoquina, Psidium firmum e Tabebuia aurea são espécies mais resistentes às queimadas e parecem ser beneficiadas com esse distúrbio para se estabelecerem, haja vista que suas densidades são superiores na área queimada. Tabebuia aurea, p. ex., apresentou 162 indivíduos a mais do que na área não queimada.

Nesse sentido, provavelmente a frequência das queimadas ocorridas no Cerrado sentido restrito no PESCAN não foi suficiente para evidenciar o efeito do fogo no processo de alteração na composição das espécies lenhosas. Entretanto, os resultados evidenciaram que o fogo pode exercer papel relevante na modificação da estrutura da vegetação, conforme ressaltado por outros autores (COUTINHO, 1990; RAMOS, 1990; SAMBUICHI, 1991).

\section{AGRADECIMENTOS}

A Glein Monteiro de Araújo e todos aqueles que, de alguma forma, contribuíram nos trabalhos de campo.

\section{REFERÊNCIAS}

ARCHER, S. et al. Savanna biodiversity and ecosystem properties - Biodiversity and savanna ecosystem processes: a global perspective. Berlin: Springer, 1996. p.207-215.

ASSUNÇÃO, S. L.; FELFILI, J. M. Fitossociologia de um fragmento de cerrado sensu stricto na APA de Paranoá, DF, Brasil. Acta Botânica Brasílica, v.18, n.4, p.903-909, 2004.

BALDUINO, A. P. C. et al. Fitossociologia e analise comparativa da composição florística do cerrado da flora de Paraopeba-MG. Revista Arvore, v.29, n.1, p.25-35, 2005.

R. Árvore, Viçosa-MG, v.33, n.4, p.695-704, 2009
BOND, W. J.; WILGEN, B. W. Fire and plants. New York: Chapman \& Hall, 1996. 263p.

BRIDGEWATER, S.; RATTER, J. A.; RIBEIRO, J. F. Biogeografic patterns, B-diversity and dominance in the cerrado biome of Brazil. Biodiversity and Conservation, v.13, n.12, p.2295-2318, 2004.

BROWER, J. E.; ZAR, J. H. Field and laboratory methods for general ecology. 2.ed. Ames: Wm. C. Brown Company, 1984. 226p.

COUTINHO, L. M. Fire in the ecology of Brasilian Cerrado. In: GOLDAMMER, J.G., (Ed.) Fire in the Tropical Biota: ecological processes and global challenges. Ecological Studies. Berlin: Springer-Verlang, 1990. p.82-105.

DURIGAN, G.; LEITÃO FILHO, H. F.; RODRIGUES, R. R. Phytosocioloy and structure of a frequently burnt cerrado vegetation in SEBrazil. Flora, v.189, n.2, p.153-160, 1994.

EITEN, G. Vegetação do cerrado. In: PINTO, M. N. (Org). Cerrado: caracterização, ocupação e perspectivas. Brasília: Edunb/SEMATEC, 1994. p.17-73.

FELFILI, J. M.; SILVA JUNIOR, M. C. Floristic composition, phytosociology and comparison of cerrado and gallery forests at Fazenda Água Limpa, Federal District, Brazil. In: FURLEY, P. A.; RATTER, J. A.; PROCTOR, J.A. (Eds.). Nature and dynamics of forest savanna boundaries. London: Chapman \& Hall, 1992. p.393-415.

FELFILI, J. M. et al. Analise comparativa da florística e fitossociologia da vegetação arbórea do cerrado sensu stricto na chapada pratinha, DF-Brasil. Acta Botanica Brasílica, v.6, n.2, p.27-46, 1993.

FELFILI, J. M.; VENTUROILI, F. Tópicos em análise de vegetação. Brasília: Universidade de Brasília, 2000. v.2. (Comunicações Técnicas Florestais, 2)

FELFILI, J. M.; SILVA JÚNIOR, M. C. (Orgs.). Biogeografia do Bioma Cerrado: estudo fitofisionômico da Chapada do Espigão Mestre do São Francisco. Brasília: Universidade de Brasília, Faculdade de Tecnologia, Departamento de Engenharia Florestal, 2001. 152p. 
FELFILI, M.F.; CARVALHO, F.A.; HAIDAR, R.F. Manual para monitoramento de parcelas permanentes nos biomas Cerrado e Pantanal. Brasília: Universidade de Brasília, 2005. 55p.

FIEDLER, N. C. et al. Efeito de incêndios florestais na estrutura e composição florística de uma área de cerrado sensu stricto na Fazenda água Limpa-DF. Revista Árvore, v.28, NUMERO, p.129-138, 2004.

GILL, A. M. Fire and the Australian flora: a review. Australian Forestry, v.38, n.1, p.4-25, 1975.

HARIDASAN, M.; ARAÚJO, G. M. Aluminiumacummulating species in two forest communities in the cerrado region of central Brazil. Forest Ecology and Management, v.24, n.1, p.15-26, 1988.

HENRIQUES, R. P. B.; HAY, J. D. V. Patterns and dynamics of plant populations. In: OLIVEIRA, P.S.; MARQUIZ, R.J. (Eds.). The Cerrados of Brazil: ecology and natural history of a Neotropical Savanna. New York: Columbia University Press, 2002. 398p.

HOFFMAN, A. W.; MOREIRA, A. G. The role of fire in population dynamicsof woody plants. In: OLIVEIRA, P.S.; MARQUIZ, R.J. (Eds.). The Cerrados of Brazil: ecology and natural history of neotropical savanna. New York: Columbia University Press, 2002. 398p.

HORN, H. S. Measurement of "overlap" in comparative ecological studies. The american Naturalist, v.100, n.9, p.419-424, 1966.

KLINK, C. A.; SOLBRING, O. T. Efeito do fogo na biodiversidade de plantas do Cerrado. In: SARMIENTO, G. \& CABIDO, M. (Eds.). Biodiversidad y funcionamento de pastizales $y$ sabanas en América Latina. LOCAL: CYTED y CIELAT, 1996. p.231-244.

KOPPEN, W. Climatologia: com um estúdio de los climas de la terra. México: Fondo de Cultura Econômica, 1948. 478p.
MAGNAGO, H., SILVA, M. T. M.; FONZAR, B. C. Vegetação. In: PROJETO

RADAMBRASIL, Folha SE.22 - Goiânia. Rio de Janeiro: 1983. p.577-636. (Levantamento de Recursos Naturais, 31).

MARIMON JÚNIOR, B. H.; HARIDASAN, M. Comparação da vegetação arbórea e características edáficas de um cerradão e um cerrado sensu stricto em áreas adjacentes sobre solo distrófico no leste de Mato Grosso, Brasil. Acta Botanica Brasilica, v. 19, n.4, p.913-926, 2005.

MATteuCCI, S. D.; COLMA, A. Metodología para el estudio de la vegetación. Washington: Secretaria General de la Organización de los Estados Americanos, 1982. 162p.

MIRANDA, H. S. et al. Queimadas de Cerrado: caracterização e impactos. In: AGUIAR, L. M. S.; CAMARGO, A. J. A. (Eds.). Cerrado: ecologia e caracterização. Planaltina: Embrapa Cerrados, 2004. p.69-123.

NASCIMENTO, A. R. T.; FELFILI, J. M.; MEIRELLES, E. M. Florística e estrutura da comunidade arbórea de um remanescente de floresta estacional decidual de encosta, Monte Alegre, GO, Brasil. Acta Botanica Brasilica, v.18, n.3, p.659-669, 2004.

NOVAES, A. S. S. et al. Pedologia. In:

PROJETO RADAMBRASIL, Folha SE. 22

- Goiânia. Rio de Janeiro: 1983. p.413-576.

(Levantamento de Recursos Naturais, 31).

RAMOS, A. E. Efeitos da queima sobre a vegetação lenhosa do Cerrado. 1990. $141 \mathrm{f}$. Dissertação (Mestrado em Ecologia) -

Universidade de Brasília, Brasília, 1990.

RATTER, J. A.; DARGIE, T. C. D. An analysis of the floristic composition of 26 cerrado areas in Brazil. Edinburg Journal of Botany, v.49, n.2, p.235-250, 1992.

RATTER, J. A.; BRIDGWATER, S.; RIBEIRO, J. F. Analysis of the floristic composition of the Brazilian cerrado vegetation III: comparison of the woody vegetation of 376 areas. Edinburg Journal of Botany, v.60, n.1, p.57-109, 2003.

R. Árvore, Viçosa-MG, v.33, n.4, p.695-704, 2009 
RIBEIRO, J. F.; WALTER, B. M. T. Fitofisionomias do bioma cerrado. In: SANO, S. M.; ALMEIDA, S. P. (Eds.). Cerrado: ambiente e flora. Planaltina: Embrapa Cerrados, 1998. p.89-166.

SAMBUICHI, R. H. R. Efeitos de longo prazo do fogo periódico sobre a fitossociologia da camada lenhosa de um Cerrado em Brasília, D.F. 1991. $144 f$. Dissertação (Mestrado em Ecologia) -

Universidade de Brasília, Brasília, 1991.

SANTOS, R. M.; VIEIRA, F. A. Analise estrutural do componente arbóreo de três áreas de cerrado em diferentes estágios de conservação no município de Três Maria, Minas Gerais, Brasil. Cerne, v.11, n.4, p.399-408, 2005.
SHEPHERD, G. J. FITOPAC 1. Manual de usuário. Campinas: Departamento de Botânica da UNICAMP, 1995. 94p.

SILVA, L. O. et al. Levantamento florístico e fitossociológico em duas áreas de cerrado sensu strictu no Parque Estadual da Serra de Caldas Novas, Goiás. Acta Botânica Brasílica, v.16, n.1, p.43-53, 2002.

SOUZA, V. C.; LORENZI, H. Botânica Sistemática - Guia ilustrado para identificação das famílias de angiosperma da flora brasileira, baseado em APG II. Nova Odessa: Plantarum, 2005. 640p. 